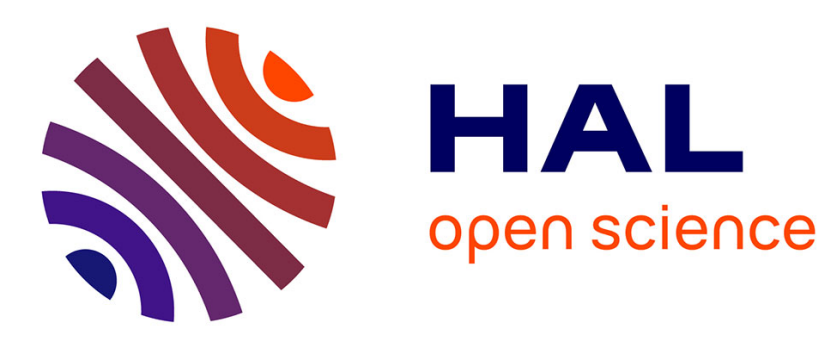

\title{
Distributed Storage in the Plane
}

Eitan Altman, Konstantin Avrachenkov, Jasper Goseling

\section{To cite this version:}

Eitan Altman, Konstantin Avrachenkov, Jasper Goseling. Distributed Storage in the Plane. IFIP Networking Conference (Networking 2014), Jun 2014, Trondheim, Norway. pp.145-153, 10.1109/IFIPNetworking.2014.6857094 . hal-01007677

\section{HAL Id: hal-01007677 https://hal.inria.fr/hal-01007677}

Submitted on 17 Jun 2014

HAL is a multi-disciplinary open access archive for the deposit and dissemination of scientific research documents, whether they are published or not. The documents may come from teaching and research institutions in France or abroad, or from public or private research centers.
L'archive ouverte pluridisciplinaire HAL, est destinée au dépôt et à la diffusion de documents scientifiques de niveau recherche, publiés ou non, émanant des établissements d'enseignement et de recherche français ou étrangers, des laboratoires publics ou privés. 


\title{
Distributed Storage in the Plane
}

\author{
Eitan Altman*, Konstantin Avrachenkov* and Jasper Goseling ${ }^{\dagger \ddagger}$ \\ * INRIA Sophia Antipolis, France \\ $\dagger$ University of Twente, The Netherlands \\ $\ddagger$ Delft University of Technology, The Netherlands \\ eitan.altman@sophia.inria.fr, k.avrachenkov@sophia.inria.fr, j.goseling@utwente.nl
}

\begin{abstract}
We consider storage devices located in the plane according to a general point process and specialize the results for the homogeneous Poisson process. A large data file is stored at the storage devices, which have limited storage capabilities. Hence, they can only store parts of the data. Clients can contact the storage devices to retrieve the data. We compare the expected cost of obtaining the complete data under uncoded as well as coded data allocation strategies. It is shown that for the general class of cost measures where the cost of retrieving data is increasing with the distance between client and storage devices, coded allocation outperforms uncoded allocation. The improvement offered by coding is quantified for two more specific classes of performance measures. Finally, our results are validated by computing the costs of the allocation strategies for the case that storage devices coincide with currently deployed mobile base stations.
\end{abstract}

\section{INTRODUCTION}

Consider wireless devices located in the plane that serve as storage devices for a big piece of data. Clients are interested in obtaining the complete data. However, the capacity of the storage devices is limited and it is not possible to store the complete data in each device. Therefore, clients will have to gather data from several devices in order to retrieve the complete piece of data.

In recent years, there has been a lot of interest- from academia [1]-[5], industry [6] and regulatory bodies [7]- in employing caches at base stations with the aim of bringing data as close as possible to the end user so as to offer a faster and better service. One of the ideas is that several base stations can provide data in a cooperative fashion. Taking this idea further has led to consideration of device-to-device connections in cellular networks where the user devices themselves act as caches [8]. Such means of content delivery provide an immediate application of the setting that was introduced above and that will be explained in more detail below. In addition to content delivery, there are a number of more specific applications of which we mention two: The first application is in the context of road traffic sensor networks. On one hand, sensors have very limited memory and transmission capabilities; on the other hand, traffic applications like provision of current traffic conditions, suggesting alternative routes or directing to empty parking spots require dealing with a large information load. Thus, it is very natural to use distributed memory storage and caching in road traffic sensor networks. The second direct application is Information Centric Networking (ICN). ICN is a new paradigm for the network architecture where the data is addressed by its name or content directly rather than by its

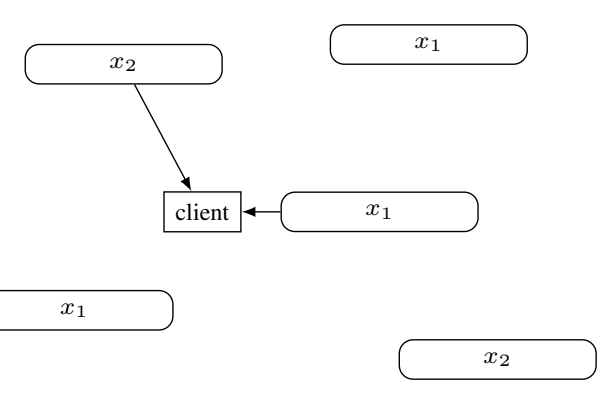

Fig. 1. Uncoded data allocation

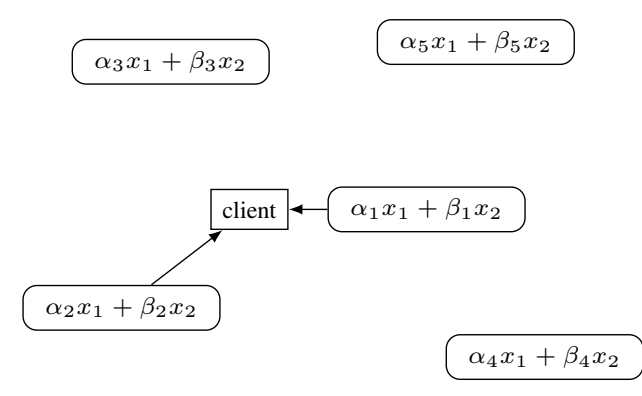

Fig. 2. Coded data allocation

physical location. There is no predefined location for the data in ICN and the content is naturally cached along the retrieval path. Examples of the ICN architecture are CCN/NDN [9], DONA [10] and TRIAD [11]. Our results can be useful for the design of the wireless networks with the ICN architecture.

Next we present our setting in more detail. We consider the very general model where storage devices are located in the plane according to a general point process and the cost of obtaining data from a particular storage device is increasing with the distance between the client and that device. This cost can reflect, for instance, the time required to obtain the data, the transmit power that needs to be used at the storage device to satisfy certain QoS constraints, or the probability with which the client fails in obtaining the data.

In this paper we study the cost resulting from two different allocation strategies for the storage devices, i.e., strategies that decide what data to store in which device. In particular, we 
will study two strategies that we will introduce next by means of an example. Suppose that the data consists of two packets, $x_{1}$ and $x_{2}$, of equal size. Assume, moreover, that the capacity of a device equals the size of a single packet.

One particular strategy that we will analyze is to store in each device either $x_{1}$ or $x_{2}$. More precisely, for each device we choose at random, independent of the other devices, to store $x_{1}$ (with probability $1 / 2$ ) or $x_{2}$ (with probability $1 / 2$ ). Now, in order to obtain all data, a client would need to contact two devices. In fact, in order to minize cost it will request $x_{1}$ from the nearest device that has $x_{1}$ and $x_{2}$ from the nearest device that has $x_{2}$. We will refer to this strategy, illustrated in Figure 1, as the uncoded strategy.

The second strategy that we consider is based on using coded data. In particular, we store at each device a random linear combination of $x_{1}$ and $x_{2}$. Now, with high probability, the linear combinations of any set of two devices will form a full rank system. Therefore, the client can contact the two closest devices and obtain sufficient information to retrieve the original data. This is illustrated in Figure 2. It is suggested by Figures 1 and 2 that the coded strategy outperforms the partitioning strategy in the sense that the distances between client and contacted devices are reduced. One of the contributions of the current work is to proof this result rigorously for a broad class of performance measures.

In addition, we will be interested in two more specific performance measures. The first is the hit rate, i.e., the probability that the client can retrieve the data from storage devices that are located within a specified range. The second performance measure is the expected total cost of retrieving the data, where total cost is the sum of costs of obtaining individual fragments. The cost of obtaining a fragment is increasing in the distance between client and storage device.

Applications of linear network coding for distributed storage were studied in [12], where it has been demonstrated that repair bandwidth can be significantly reduced. In the current work there is no notion of repair. Instead, coding is used to bring the data 'closer' to the client. The use of coding was also explored in [13] where it was shown how to efficiently allocate the data at caches with the aim of ensuring that any sufficiently large subset of caches can provide the complete data. The difference with the current work is that we are taking the geometry of the deployment of the storage devices into account. In [14] coding strategies for networks of caches are presented, where each user has access to a single cache and a direct link to the source. It is demonstrated how coding helps to reduce the load on the link between the caches and the source. Note that we assume that different transmissions from caches to the clients are orthogonal, for instance by separating them in time or frequency. In [15] the impact of non-orthogonal transmissions is considered and scaling results are derived on the best achievable transmission rates. Systems of distributed storage devices or caches can be classified according to the amount of coordination between the devices. In [16] an approach with implicit coordination is proposed. Networks of caches are notoriously difficult to analyze. Only some very particular topologies and caching strategies (see [17] and references therein) or approximations [18], [19] have been studied. In a recent work [20] ergodicity of cache networks has been investigated. Using continuous geometrical constraints on cache placement instead of combinatorial constraints allows us to obtain exact analytical results.

Other work on caching in wireless networks is, for instance, [4], [5], [21]-[23]. In [21] the authors analyze the trade-off between energy consumption and the retrieval delay of data from the caches. In [22], the authors consider the optimal number of replicas of data such that the distance between a requesting node and the nearest replica is minimized. Data sharing among multiple caches such that the bandwidth consumption and the data retrieval delay are minimal is considered in [23]. None of [4], [5], [21]-[23] are considering coded caching strategies.

We would like to emphasize that none of the above mentioned works considered continuous geometric constraints on storage device placement. Thus, to the best of our knowledge, the current paper is the first work on the analysis of spatial distributed storage with stochastic geometry. In the remainder we will often refer to the storage devices as caches. Note, however, that the current work is dealing with distributed storage of a single file and that we do not consider dynamic caching strategies for multiple files.

The contributions of the current work are as follows:

- We consider an arbitrary placement of the caches in the plane and an arbitrary increasing performance measure. We show that network coding outperforms partitioning for all values of the field size used in the coding.

- For caches placed according to a homogeneous Poisson process we provide closed form expressions for the waiting time and the outage probability for both the coded and the partioned placement strategy.

- For caches placed according to a homogeneous Poisson process we provide an upper bound on the ratio of the waiting time of the partioned strategy and the waiting time of the coded strategy.

- We evaluate the waiting time of the coded and partioned strategy for cache positions that correspond to the locations of $3 \mathrm{G}$ base stations as provided by the OpenMobileNetwork project [24]. We show that the insights obtained from our analytical results also hold in this setting.

The remainder of this paper is organized as follows. In Section II we define the model and formulate and exact problem statement. Our main theoretical results are given in Section III. In Section IV we provide numerical examples. In particular, we consider network topologies generated by spatial Poisson process and from a real wireless network. We conclude and give future research directions in Section V.

\section{Model AND PROBlem StATEMENT}

\section{A. System}

We consider storage devices that are positioned in the plane and enumerate the devices with respect to their distance to the 
client starting with device closest to the client. The indices of the devices are denoted as $I_{1}, I_{2}, \ldots$ and we denote by $D(I)$ the distance between the client and the $I$-th device. For a vector $\mathbf{I}=\left(I_{1}, I_{2}, \ldots\right)$, let $D(\mathbf{I})=\left(D\left(I_{1}\right), D\left(I_{2}\right), \ldots\right)$. In the remainder we consider two scenarios: i) the storage devices are placed at arbitrary positions and we derive results that hold for any such placement, ii) devices are positioned according to a homogeneous Poisson process (HPP) with intensity $\lambda$. In the second scenario $D(I)$ is a random variable and we will derive results that hold in expectation.

The data $M$ consists of $k$ symbols, where each symbol is selected uniformly at random from $\{1, \ldots, q\}$, i.e.,

$$
M=\left(M_{1}, \ldots, M_{k}\right) .
$$

A particular instance of such a $q$-ary symbol is that of a tuple of many bits. Each device has the capacity to store one $q$-ary symbol. There are various approaches to placing the message in the device. Analyzing these placement strategies is the main problem studied in this paper. We introduce the strategies in Section II-B.

A client, located at an arbitrary location in the plane, needs to retrieve $M$ by combining information from various devices. We assume that the client has full knowledge of the locations of the devices as well as the placement strategy that has been used. Clients can request data from devices. The cost of obtaining the data from devices is increasing with the distance between the client and these devices. A client will have to contact several devices to obtain the complete message.

Our interest is in the expected cost, where the expectation is over the randomness in the placement strategy as well as over the location of the devices in case of placement according to a Poisson process.

\section{B. Strategies}

We will consider two strategies.

1) Uncoded: In the first strategy that we consider we simply store one of the symbols $M_{1}, \ldots, M_{k}$ in a device. This is done independently and at random for each device by choosing for each device a label uniformly at random from $\{1, \ldots, k\}$. In a device with label $t$ we store $M_{t}$.

In the delivery phase the client contacts for each $t \in$ $\{1, \ldots, k\}$ the closest device with that label and retrieves $M_{t}$. We denote by $I_{1}^{p}<I_{2}^{p}<\cdots<I_{k}^{p}$ the index of the device from which the parts are obtained, i.e., if $I_{i}^{p}=j$, then the $i$-th part is obtained from the $j$-th nearest device. Let $\mathbf{I}^{p}=\left(I_{1}^{p}, \ldots, I_{k}^{p}\right)$.

2) Random linear coding: The second strategy is based on random linear coding. We interpret $M_{1}, \ldots, M_{k}$ as symbols over the field $\mathbb{F}_{q}$. For each device we draw coefficients $c_{i}$, $i=1, \ldots, k$, independently and uniformly at random from $\mathbb{F}_{q}$. In the device we store

$$
\sum_{i=1}^{k} c_{i} M_{i} .
$$

In the delivery phase we retrieve from the set of $k$ nearest devices that provide complete data. In particular, the first part is obtained from the first neighbor. If the second neighbor has a linear combination that is linearly independent from the part of the first neighbor it is retrieved. Otherwise, we check the content of the third neighbor, etc. Let $I_{1}^{c}<I_{2}^{c}<\cdots<$ $I_{k}^{c}$ denote indices of the neighbors from which the parts are obtained. Let $\mathbf{I}^{c}=\left(I_{1}^{c}, \ldots, I_{k}^{c}\right)$.

\section{Performance measures}

We have defined two caching strategies. In both strategies the client retrieves the data from exactly $k$ devices. We express performance of the system in terms of the expected cost of contacting these devices. The cost is dependent on the distance from which the data is obtained. Let $\delta_{1}, \ldots, \delta_{k}$ denote these distances. Using the notation from Section II-B we have $\delta_{i}=$ $D\left(I_{i}^{p}\right)$ and $\delta_{i}=D\left(I_{i}^{c}\right)$ in the uncoded and coded strategy respectively.

In the remainder we will present results for a general cost measure $G: \mathbb{R}_{+}^{k} \rightarrow \mathbb{R}_{+}$, where we used $\mathbb{R}_{+}=[0, \infty)$. In addition we will give results for a two classes of more specific cost measures. These classes will be introduced in the subsequent subsections. First we give the constraints that we impose on all the cost measures that we consider. We assume that $G$ is an increasing function in the sense that $\delta_{i} \geq \tilde{\delta}_{i}$ for all $i=1, \ldots, k$ implies that $G\left(\delta_{1}, \ldots, \delta_{k}\right) \geq G\left(\tilde{\delta}_{1}, \ldots, \tilde{\delta}_{k}\right)$. In addition $G$ is assumed to be bounded by $G_{\max }$, i.e., $0 \leq G\left(\delta_{1}, \ldots, \delta_{k}\right) \leq G_{\max }$ for all $\left(\delta_{1}, \ldots, \delta_{k}\right) \in \mathbb{R}_{+}^{k}$. The rationale of the upper bound is that if the devices that provide complete data are located too far away a client can obtain the data directly from the source using some other technology.

1) Energy consumption, waiting time, etc.: We introduce the cost function

$$
W\left(\delta_{1}, \ldots, \delta_{k}\right)=\sum_{i=1}^{k} \min \left\{\left(\delta_{i}\right)^{a},\left(\delta_{\max }\right)^{a}\right\},
$$

where $a>0$ is a parameter.

This cost function can be related to various quantities of interest. For instance, it can denote the energy consumed by a device in order to successfully deliver data to a client. Based on

$$
R=\frac{1}{2} \log \left(1+P \delta^{-a}\right),
$$

where $a$ is the path loss exponent of the wireless medium, we see that the transmit power $P$ required to transmit at a guaranteed minimum rate $R$ satisfies $P=(\exp (2 R)-1) \delta^{a}$. Therefore, the energy required to deliver a symbol from the device is of the form (3), with preconstant proportional to $\exp (2 R)-1$. In this case $a$ denotes the path loss exponent, which in the plane satisfies $a \geq 2$.

Another example is given by the time required to obtain the data if TCP is used. The throughput of TCP is known to be inversely proportional to the round trip delay, $c f$. [25] or [26]. The later is given by the sum of the processing/queueing delay (which we assume zero) and the propagation delay, which is proportional to the distance $\delta$. Therefore, $W=C \delta$, i.e., $a=1$, gives the time required to obtain data from a device using TCP. 
2) Cache miss probability: We consider the case that clients can only connect to devices within range $r$. If the complete data cannot be retrieved from the devices within this range, a cache miss occurs. If clients can connect to any cache within range $r$, but not outside, then $\mathbb{E}[F(D(\mathbf{I})]$, with

$$
F\left(\delta_{1}, \ldots, \delta_{k}\right)= \begin{cases}0, & \text { if } \max _{i} \delta_{i} \leq r, \\ 1, & \text { otherwise, }\end{cases}
$$

denotes the probability that data can not be obtained successfully.

\section{RESULTS}

\section{A. General cost measures}

Observe that the random linear coding strategy depends on the client to find a sufficient number of linearly independent combinations in devices that are not too far away. If $q$ is small there is a significant probability that the information of the next device is not linearly independent. Hence, one might argue that for small values of $q$ there exists performance measures for which the partitioning strategy outperforms random linear coding. Our first result demonstrates that this is not true, i.e., that random linear coding outperforms the partitioning strategy for any value of $q$ and any cost function.

Theorem 1. Consider an arbitrary placement of devices, a bounded increasing function $G$ and any $q$. Then

$$
\mathbb{E}\left[G\left(D\left(\mathbf{I}^{c}\right)\right)\right] \leq \mathbb{E}\left[G\left(D\left(\mathbf{I}^{p}\right)\right)\right],
$$

i.e., coding always outperforms partitioning.

The proof of the above theorem is given in Appendix A. Note, that the above result in particular implies that coding is better than uncoded strategy for any realization in case of placement according to a spatial Poisson process.

Since less than $k$ devices can never provide the complete data, it is clear that the minimum cost that one can hope to achieve is given by the cost of contacting the nearest $k$ devices. For notation convenience, denote the minimum expected cost as

$$
G_{\min }=\mathbb{E}[G(D(1), \ldots, D(k))] .
$$

Our next result provides a bound on the deviation of the cost of the coded strategy from $G_{\min }$.

Theorem 2. The expected cost of the coded strategy is upper bounded as

$$
\mathbb{E}\left[G\left(D\left(\mathbf{I}^{c}\right)\right)\right] \leq G_{\min }+G_{0},
$$

where $G_{0}=G_{\max }\left(1-(1-1 / q)^{k}\right)$.

Proof: Let $A$ denote the event that the first $k$ devices provide a full rank system. Then

$$
\begin{aligned}
& \mathbb{E}\left[G\left(D\left(\mathbf{I}^{c}\right)\right)\right]=\mathbb{E}\left[G\left(D\left(\mathbf{I}^{c}\right)\right) \mid A\right] P(A) \\
&+ \mathbb{E}\left[G\left(D\left(\mathbf{I}^{c}\right)\right) \mid \bar{A}\right] P(\bar{A})
\end{aligned}
$$

and the result follows from the assumption that $G$ is bounded by $G_{\max }$ and $P(\bar{A}) \leq 1-(1-1 / q)^{k}, c f$. [27] or [28].
We will illustrate the above result for a specific performance measure below. In particular, we will show that the coded strategy is close to optimal.

\section{B. Waiting time}

Next, we consider specific instances of the cost. First we consider $W$ as defined in Section II-C1. Before giving the results we will provide some definitions and results on the Gamma function. Let $\Gamma(k, x)$ denote the upper incomplete Gamma function, i.e.,

$$
\Gamma(k, x)=\int_{x}^{\infty} z^{k-1} e^{-z} d z .
$$

Furthermore, we define $\Gamma(k)=\Gamma(k, 0)$ and $\gamma(k, x)=\Gamma(k)-$ $\Gamma(k, x)$. The use of $\Gamma(k, x)$ for Poisson processes stems from the fact that for a Poisson random variable $X$ with mean $\mu$ we have

$$
P(X<n)=e^{-\mu} \sum_{i=0}^{n-1} \frac{\mu^{i}}{\Gamma(i+1)}=\frac{\Gamma(n, \mu)}{\Gamma(n)} .
$$

Therefore, the probability that $D_{n}$, the distance to the $n$-th nearest neigbor in an HPP of density $\lambda$ is at most $\delta$ is

$$
P\left(D_{n} \leq \delta\right)=1-\frac{\Gamma\left(n, \lambda \pi \delta^{2}\right)}{\Gamma(n)} .
$$

We are now ready to present our first result on $W$. The proof of the next theorem is given in Appendix B.

Theorem 3. Let the devices be distributed in the plane as HPP with density $\lambda$. Then, the expected costs of the partitioning and coded strategies are

$$
\begin{aligned}
W^{p} & =k\left(\frac{k}{\lambda \pi}\right)^{b-1} \gamma\left(b, \frac{d}{k}\right)+k\left(\frac{d}{\lambda \pi}\right)^{b-1} \Gamma\left(1, \frac{d}{k}\right), \\
W_{\min }^{c} & =\frac{\gamma(k+b, d)+b d^{b-1} \Gamma(k+1, d)-(b-1) d^{b} \Gamma(k, d)}{(\lambda \pi)^{b-1} b \Gamma(k)},
\end{aligned}
$$

where $b=a / 2+1$ and $d=\lambda \pi \delta_{\max }^{2}$.

To get an idea about the nature of the above involved equations, a reader may check Figure 4 where a numerical example is presented.

From the observation that

$$
\lim _{d \rightarrow \infty} d^{c} \Gamma(k, d)=0,
$$

for any $c$, we immediately obtain the following corollary which provides limiting expressions for $W^{p}$ and $W_{\min }^{c}$ for $\delta_{\max } \rightarrow$ $\infty$.

Corollary 1. Let $\bar{W}^{p}=\lim _{\delta_{\max } \rightarrow \infty} W^{p}$ and $\bar{W}_{\min }^{c}=$ $\lim _{\delta_{\max } \rightarrow \infty} W_{\min }^{c}$. We have

$$
\begin{gathered}
\bar{W}^{p}=k\left(\frac{k}{\lambda \pi}\right)^{a / 2} \Gamma\left(1+\frac{a}{2}\right), \\
\bar{W}_{\min }^{c}=\frac{\Gamma\left(k+1+\frac{a}{2}\right)}{\left(1+\frac{a}{2}\right)(\lambda \pi)^{a / 2} \Gamma(k)} .
\end{gathered}
$$


Next we turn our attention to the cost benefit of coding over partitioning, defined as the ratio $\bar{W}^{p} / \bar{W}_{\min }^{c}$. Clearly, it is an optimistic prediction. However, in the numerical examples section we shall demonstrate that the actual gain is typically close to this optimistic prediction. It follows readily from Corollary 1 that

$$
\frac{\bar{W}^{p}}{\bar{W}_{\min }^{c}}=\left(1+\frac{a}{2}\right) k^{1+a / 2} B\left(k, 1+\frac{a}{2}\right),
$$

where $B\left(k, 1+\frac{a}{2}\right)$ denotes the Beta function.

Theorem 4. The benefit of coding over partitioning, $\bar{W}^{p} / \bar{W}_{\text {min }}^{c}$, is increasing in $k$. Moreover

$$
\lim _{k \rightarrow \infty} \frac{\bar{W}^{p}}{\bar{W}_{\min }^{c}}=\left(\frac{a}{2}+1\right) \Gamma\left(\frac{a}{2}+1\right) .
$$

Hence

$$
1 \leq \frac{W^{p}}{W^{c}} \leq\left(\frac{a}{2}+1\right) \Gamma\left(\frac{a}{2}+1\right),
$$

with equality on the LHS iff $k=1$.

Proof: We start with proving that $\bar{W}^{p} / \bar{W}_{\text {min }}^{c}$, is increasing in $k$. Let $b=1+\frac{a}{2}$. We have

$$
\frac{\partial}{\partial k} \frac{\bar{W}^{p}}{\bar{W}_{\min }^{c}}=b k^{b} B(k, b)\left[\frac{b}{k}+\psi(k)-\psi(k+b)\right],
$$

where $\psi(x)=\int_{0}^{\infty}\left(\frac{e^{-t}}{t}-\frac{e^{-x t}}{1-e^{-t}}\right) d t$ is the digamma function [29]. We need to show that

$$
\int_{0}^{\infty} e^{-k t} \frac{1-e^{-b t}}{1-e^{-t}} d t \leq \frac{b}{k},
$$

but this follows directly from the observation that $\frac{1-e^{-b t}}{1-e^{-t}} \leq b$.

The limiting expression follows from an application of Stirlings approximation.

Note, that for $a=1,2,3$, the upper bound in Theorem 4 reduces to $\frac{3 \sqrt{\pi}}{4} \approx 1.3,2$ and $\frac{15 \sqrt{\pi}}{8} \approx 3.3$ respectively.

\section{Cache miss probability}

Next let us consider the cache miss probability.

Theorem 5. The cache miss probability of the partitioning and coded strategy are

$$
\begin{aligned}
F^{p} & =1-\left(1-e^{-\lambda \pi r^{2} / k}\right)^{k}, \\
F_{\min }^{c} & =\frac{\Gamma\left(k, \lambda \pi r^{2}\right)}{\Gamma(k)} .
\end{aligned}
$$

Proof: For the coded strategy we have

$$
F_{\text {min }}^{c}=P(D(k)>r)=\frac{\Gamma\left(k, \lambda \pi r^{2}\right)}{\Gamma(k)},
$$

since the $k$-th nearest cache needs to be within distance $r$.

For the uncoded strategy we have

$$
\begin{aligned}
F^{p} & =1-P_{\lambda / k}\left(D_{1} \leq r\right)^{k} \\
& =1-\left(1-e^{-\lambda \pi r^{2} / k}\right)^{k},
\end{aligned}
$$

which follows from the fact that each of the parts is found in the first neighbour in a (thinned) Poisson process of intensity $\lambda / k$.

We note that the following asymptotics for equations in the statement of Theorem 5 take place

$$
\begin{gathered}
F^{p} \approx 1-\frac{\left(\lambda \pi r^{2}\right)^{k}}{k^{k}}, \\
F_{\text {min }}^{c} \approx 1-e^{-\lambda \pi r^{2}} \frac{\left(\lambda \pi r^{2}\right)^{k}}{k !},
\end{gathered}
$$

for large values of $k$. This indicates that when increasing $k$, the cache miss probability increases much faster for the uncoded strategy than for the coded strategy. We shall illustrate this phenomenon with a specific numerical example in the next section.

\section{Evaluation}

Let us evaluate our theoretical results for distributed memory devices placed according to (a) Spatial Homogeneous Poisson Process and (b) real wireless network. In order to compare these two settings we have chosen the intensity of the Poisson process equal to the density of base stations in the real network. In Figure 3 one can see a realization of the Spatial Homogeneous Poisson Process with $\lambda=1.8324 \times 10^{-5}$. The value is rather small because we measure distances in meters. In Figure 4 we have plotted $W^{p}, W_{\min }^{c}$ and $W_{\min }^{c}+W_{0}$ given by formulae of Theorems 2 and 3 as functions of the number of parts in which a file is divided for $q=2^{8}, a=2$ and $\delta_{\max }=700$. On this figure we have also plotted the averaged cost functions for uncoded and coded strategies from the simulation of the system. We have averaged over 500 realizations of the Poisson process and 100 realizations of the part distribution. The simulation curve for the uncoded strategy follows very closely the theoretical result. Of course, this is no surprise if the simulations and theoretical derivations have been done correctly. What is more interesting is that the simulation curve for the coded strategy follows very closely the lower bound given by $W_{m i n}^{c}$. This can be explained by the fact that when the number of parts $k$ is not large, the event of obtaining not a full rank system after $k$ requests to devices is very small.

Next we evaluate the performance of coded and uncoded strategies on the topology of a real wireless network. We have taken the positions of $3 \mathrm{G}$ base stations provided by the OpenMobileNetwork project [24]. The base stations are situated in the area $1.95 \times 1.74 \mathrm{kms}$ around the TU-Berlin campus. One can see the positions of the base stations from the OpenMobileNetwork project in Figure 5. We note that the base stations of the real network are more clustered because they are typically situated along the roads. In Figure 6 we have plotted the averaged simulation results for coded and uncoded strategies for the real network topology and Poisson process with the intensity equal to the density of the base stations. The averaging for the real network data has been done with respect to the user position and part placement. In order to avoid border effect we have placed a user in a smaller centered 


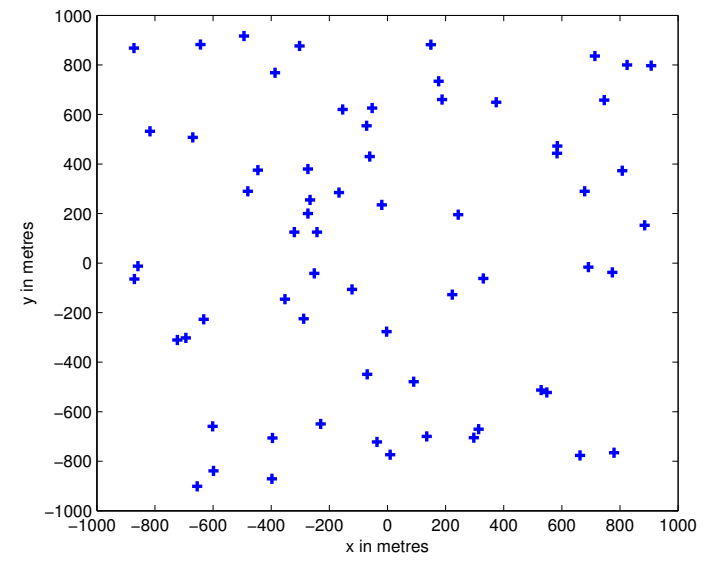

Fig. 3. A realization of the Spatial Homogeneous Poisson Process.

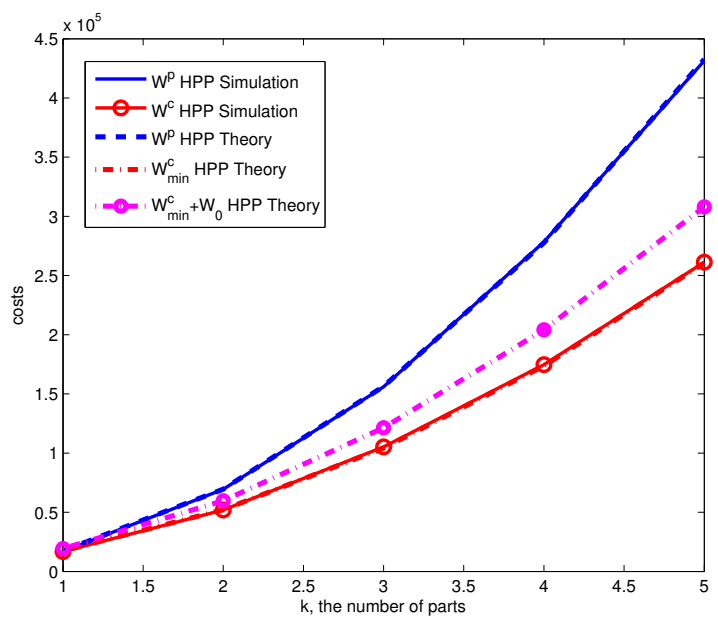

Fig. 4. Cost function, bounds and simulation results for Poisson process.

rectangular region with the lengths of the sides equal to half of the respective sides. We observe that the clustering actually decreases the cost and the decrease in the cost of the uncoded strategy is larger than the decrease in the cost of the coded strategy. A possible intuitive explanation for this phenomenon is that for uncoded strategy it is difficult to find the last parts and clustering helps to reduce the marginal cost of doing more trials at the end of the discovery process.

For the same $\lambda=1.8324 \times 10^{-5}$ and $r=700$ we use the formulae from Theorem 5 to plot the miss probabilities $F^{p}$ and $F_{\text {min }}^{c}$ in Figure IV. Suppose one wants the miss probability to be not larger than $10^{-2}$. Then, for the uncoded strategy one should not divide the file into more than 5 parts, whereas for the coded strategy one can have up to 17 parts. This in turn implies that the required storage capacity is smaller under the coded strategy. This highlights another benefit, flexibility, of the coded strategy for spatial distributed storage.

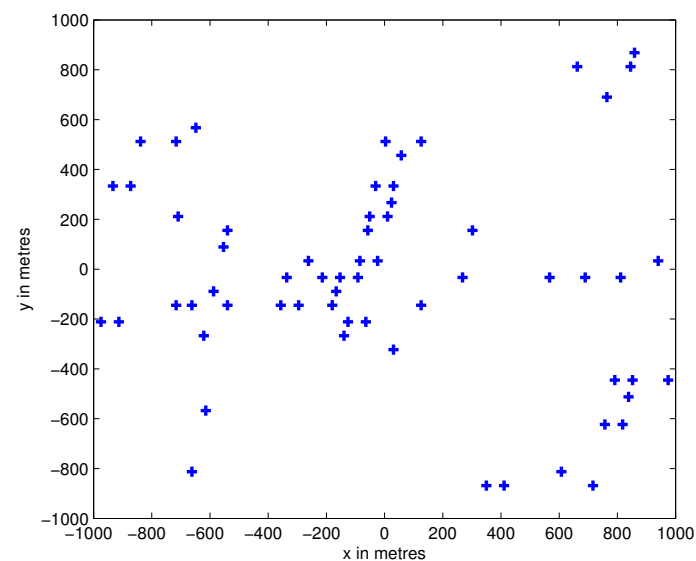

Fig. 5. Location of Base Stations from OpenMobileNetwork dataset.

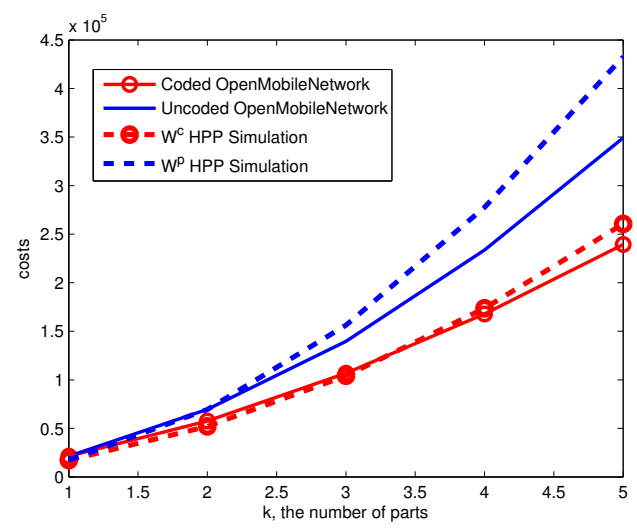

Fig. 6. Comparison OpenMobileNetwork versus Poisson process.

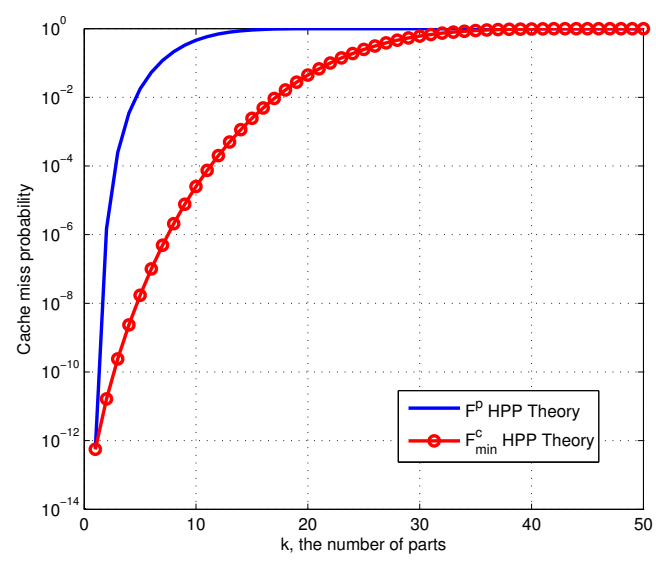

Fig. 7. Cache miss probability for Poisson process. 


\section{Discussion}

In this paper we have analyzed allocation strategies for distributed storage in a network of wireless devices. In particular, we have studied coded and uncoded deployment and allocation strategies for a single client that is requesting all data. In future work we will focus on the dynamic setting in which there are many clients that are requesting data over time. Hence, we obtain a spatial queueing model. One particular problem to study in this scenario is the stability of such a system if we take into account the interference caused by multiple caches transmitting simultaneously. Another problem is on actual caching strategies if clients request only part of the data. In particular, if there are many different pieces of data with varying popularity, the challenge is to design distributed coded caching strategies that minimize overall cost, i.e., maximize hit rate.

\section{ACKNOWLEDGEMENT}

Part of this work was done while J. Goseling was visiting the MAESTRO group at INRIA Sophia Antipolis, France, in November 2011. The hospitality and support provided by INRIA are greatly acknowledged. This work was supported in part by the Netherlands Organization for Scientific Research (NWO) grant 612.001.107, by Labex "Laboratoire dExcellence" UCN, by Orange Labs grant on caching systems and by the European Commission within the framework of the CONGAS project FP7-ICT-2011-8-317672.

\section{APPENDIX A}

\section{PROOF OF THEOREM 1}

Observe that Theorem 1 provides a stochastic comparison result. To simplify discussion in the remainder we introduce for random vectors $\mathbf{Z}=\left(Z_{1}, \ldots, Z_{k}\right)$ and $\tilde{\mathbf{Z}}=\left(\tilde{Z}_{1}, \ldots, \tilde{Z}_{k}\right)$ the notation $\mathbf{Z} \leq$ st $\tilde{\mathbf{Z}}$, to denote that $\mathbb{E}[F(\mathbf{Z})] \leq \mathbb{E}[F(\tilde{\mathbf{Z}})]$ for all bounded increasing $F$. We say that $\mathbf{Z}$ is less than $\tilde{\mathbf{Z}}$ in the usual stochastic ordering.

Lemma 1. $\mathbf{I}^{c} \leq_{\mathrm{st}} \mathbf{I}^{p}$.

Proof: We will make use of a result by Veinott [30], $c f$. [31, Theorem 3.3.7] that states that the result holds if $I_{1}^{c} \leq_{\mathrm{st}} I_{1}^{p}$ and for $i=2,3, \ldots, k$,

$$
\begin{aligned}
& {\left[I_{i}^{c} \mid I_{1}^{c}=x_{1}^{c}, \ldots, I_{i-1}^{c}=x_{i-1}^{c}\right] \leq_{\mathrm{st}}} \\
& \quad\left[I_{i}^{p} \mid I_{1}^{p}=x_{1}^{p}, \ldots, I_{i-1}^{p}=x_{i-1}^{p}\right],
\end{aligned}
$$

whenever $x_{j}^{c} \leq x_{j}^{p}$ for all $j=1, \ldots, i-1$.

We have $I_{1}^{c}=1$ and for $i=2, \ldots, k$

$$
\left[I_{i}^{c} \mid I_{1}^{c}=x_{1}^{c}, \ldots, I_{i-1}^{c}=x_{i-1}^{c}\right]=G_{i}+x_{i-1}^{c},
$$

where $P\left(G_{i}=n\right)=\left(1-g_{i}\right) g_{i}^{n-1}, g_{i}=q^{i-1} / q^{k}$, i.e., $G_{i}$ is geometrically distributed with parameter $g_{i}$. The above follows from the fact the coding coefficients are chosen independently for each cache. The probability that the vector of coefficients $\left(c_{1}, \ldots, c_{k}\right)$ is linearly dependent of the $i-1$ vectors that have previously been obtained is $q^{i-1} / q^{k}$, where $q^{k}$ is the total number of possible vectors from which one is selected uniformly at random, and $q^{i-1}$ is the number of vectors that are linearly dependent.

For the partitioning strategy we have $I_{1}^{p}=1$ and

$$
\left[I_{i}^{p} \mid I_{1}^{p}=x_{1}^{p}, \ldots, I_{i-1}^{p}=x_{i-1}^{p}\right]=\tilde{G}_{i}+x_{i-1}^{p},
$$

where $P\left(\tilde{G}_{i}=n\right)=\left(1-\tilde{g}_{i}\right) \tilde{g}_{i}^{n-1}, \tilde{g}_{i}=(i-1) / k$, which follows from the observation that there are $k$ different symbols that could be obtained, of which $i-1$ are not useful.

Now observe that $g_{i}<\tilde{g}_{i}$ for all $q$, all $k$, and all $i=$ $2, \ldots, k$. Therefore, $P\left(G_{i} \leq z\right) \geq P\left(\tilde{G}_{i} \leq z\right)$ for all $z$ and $G_{i} \leq_{\text {st }} \tilde{G}_{i}, c f$. [31, Theorem 1.2.8]. Finally, note that $G_{i} \leq_{\text {st }}$ $\tilde{G}_{i}$ implies that $G_{i}+x_{i-1}^{c} \leq_{\text {st }} \tilde{G}_{i}+x_{i-1}^{p}$ whenever $x_{i-1}^{c} \leq$ $x_{i-1}^{p}$.

The proof of Theorem 1 follows directly from the above lemma.

\section{APPENDIX B}

\section{PROOF OF THEOREM 3}

We first give some technical lemmas.

\section{Lemma 2.}

$$
\int_{x}^{\infty} z^{c} \Gamma(k, z) d z=\frac{\Gamma(k+c+1, x)-x^{1+c} \Gamma(k, x)}{c+1} .
$$

Proof: For $k=1$, (28) follows from $\Gamma(1, z)=e^{-z}$ and an integration by parts. We proceed by induction over $k$. By repeatedly using the recurrence identity

$$
\Gamma(k, x)=(k-1) \Gamma(k-1, x)+x^{k-1} e^{-x},
$$

which is easily shown using an integration by parts, and assuming that (28) holds for $k-1$ we have

$$
\begin{aligned}
\int_{x}^{\infty} z^{c} \Gamma(k, z) d z=\int_{x}^{\infty} z^{c+k-1} e^{-z} d z \\
=\int_{x}^{\infty}\left[z^{k+c-1} e^{-z}+(k-1) z^{c} \Gamma(k-1, z)\right] d z \\
=\frac{(k+c) \Gamma(k+c, x)-x^{c+1}(k-1) \Gamma(k-1, x)}{c+1} \\
=\frac{1}{c+1}\{\Gamma(k+c+1, x) \\
=\frac{\left.-x^{k+c} e^{-x}-x^{c+1}\left[\Gamma(k, x)-x^{k-1} e^{-x}\right]\right\}}{c+1} . \\
=\frac{\Gamma(k+c+1, x)-x^{1+c} \Gamma(k, x)}{c+1} .
\end{aligned}
$$

Lemma 3. For $k \in \mathbb{N}, c \geq 0$ and $x \geq 0$

$$
\begin{aligned}
\sum_{i=1}^{k} \frac{\Gamma(i+c)}{\Gamma(i)} & =\frac{\Gamma(k+c+1)}{(c+1) \Gamma(k)}, \\
\sum_{i=1}^{k} \frac{\Gamma(i+c, x)}{\Gamma(i)} & =\frac{\Gamma(k+c+1, x)-x^{c+1} \Gamma(k, x)}{(c+1) \Gamma(k)}, \\
\sum_{i=1}^{k} \frac{\gamma(i+c, x)}{\Gamma(i)} & =\frac{\gamma(k+c+1, x)+x^{c+1} \Gamma(k, x)}{(c+1) \Gamma(k)},
\end{aligned}
$$


Proof: We have

$$
\begin{aligned}
\sum_{i=1}^{k} \frac{\Gamma(i+c, x)}{\Gamma(i)} & =\int_{x}^{\infty} \sum_{i=1}^{k} \frac{z^{c+i-1}}{\Gamma(i)} e^{-z} d z \\
& =\int_{x}^{\infty} z^{c} \sum_{j=0}^{k-1} \frac{z^{j}}{\Gamma(j+1)} e^{-z} d z \\
& =\int_{x}^{\infty} z^{c} \frac{\Gamma(k, z)}{\Gamma(k)} d z \\
& =\frac{\Gamma(k+c+1, x)-x^{c+1} \Gamma(k, x)}{(c+1) \Gamma(k)},
\end{aligned}
$$

where the last equality follows from Lemma 2 . For $x=0$ this reduces to

$$
\sum_{i=1}^{k} \frac{\Gamma(i+c)}{\Gamma(i)}=\frac{\Gamma(k+c+1)}{(c+1) \Gamma(k)} .
$$

\section{Lemma 4.}

$$
\int_{0}^{u} x^{a} d\left(1-\frac{\Gamma\left(i, b x^{2}\right)}{\Gamma(i)}\right)=\frac{\gamma\left(\frac{a}{2}+i, b u^{2}\right)}{\Gamma(i) b^{a / 2}} .
$$

Proof. The result follows directly from the definitions of $\Gamma\left(i, b x^{2}\right)$ and $\gamma\left(a / 2+i, b u^{2}\right)$.

Before giving the proofs for the two strategies, recall that $b=1+a / 2$ and $d=\lambda \pi \delta_{\max }^{2}$. This leads to $\delta_{\max }^{a}=$ $(d / \lambda / \pi)^{b-1}$ and $P\left(D(i)>\delta_{\max }\right)=\Gamma(i, d) / \Gamma(i)$.

\section{A. Uncoded strategy}

The first thing to note in the uncoded strategy is that for each of the labels $t \in\{1, \ldots, k\}$ all caches with label $t$ form a homogeneous spatial Poisson process with intensity $\lambda / k$. This follows from the fact that these caches form a thinned Poisson process [32].

Now, for the uncoded strategy we have

$$
\begin{aligned}
W^{p} & =\sum_{i=1}^{k} \int_{0}^{\infty} \max \left\{\left(\delta_{i}\right)^{a}, \delta_{\max }^{a}\right\} d P\left(D\left(X_{i}^{p}\right) \leq \delta_{i}\right) \\
& =k \int_{0}^{\delta_{\max }} \delta^{a} d\left(1-\frac{\Gamma\left(1, \frac{\lambda}{k} \pi \delta^{2}\right)}{\Gamma(1)}\right)+k \delta_{\max }^{a} \Gamma\left(1, \frac{d}{k}\right) \\
& =k\left(\frac{k}{\lambda \pi}\right)^{a / 2} \gamma\left(1+\frac{a}{2}, \frac{d}{k}\right)+k\left(\frac{d}{\lambda \pi}\right)^{a / 2} \Gamma\left(1, \frac{d}{k}\right),
\end{aligned}
$$

where we have used Lemma 4.

\section{B. Random linear coding strategy}

For the coded strategy we start with

$$
W_{\min }^{c}=\sum_{i=1}^{k} \int_{0}^{\delta_{\max }} \delta^{a} d P(D(i) \leq \delta)+\delta_{\max }^{a} \sum_{i=1}^{k} \frac{\Gamma(i, d)}{\Gamma(i)}
$$

where we continue with analyzing each term seperately. First,

$$
\delta_{\max }^{a} \sum_{i=1}^{k} \frac{\Gamma(i, d)}{\Gamma(i)}=\left(\frac{d}{\lambda \pi}\right)^{a / 2} \frac{\Gamma(k+1, d)-d \Gamma(k, d)}{\Gamma(k)},
$$

by Lemma 3. Next,

$$
\int_{0}^{\delta_{\max }} \delta^{a} d P(D(i) \leq \delta)=\frac{\gamma\left(\frac{a}{2}+i, d\right)}{\Gamma(i)(\lambda \pi)^{a / 2}},
$$

by Lemma 4 and

$$
\sum_{i=1}^{k} \frac{\gamma\left(\frac{a}{2}+i, d\right)}{\Gamma(i)(\lambda \pi)^{a / 2}}=\frac{\gamma\left(k+1+\frac{a}{2}, d\right)+d^{a / 2+1} \Gamma(k, d)}{(\lambda \pi)^{a / 2}\left(\frac{a}{2}+1\right) \Gamma(k)},
$$

by Lemma 3. The result now follows from (45) and (47).

\section{REFERENCES}

[1] N. Golrezaei, A. Molisch, A. Dimakis, and G. Caire, "Femtocaching and device-to-device collaboration: A new architecture for wireless video distribution," Communications Magazine, IEEE, vol. 51, no. 4, pp. 142149, April 2013.

[2] B. Ahlgren, P. A. Aranda, P. Chemouil, S. Oueslati, L. M. Correia, H. Karl, M. Sollner, and A. Welin, "Content, connectivity, and cloud: ingredients for the network of the future," Communications Magazine, IEEE, vol. 49, no. 7, pp. 62-70, 2011.

[3] E. Bastug, J.-L. Guenego, and M. Debbah, "Cloud storage for small cell networks," in Cloud Networking (CLOUDNET), 2012 IEEE 1st International Conference on, Nov 2012, pp. 100-104.

[4] N. Abedini and S. Shakkottai, "Content caching and scheduling in wireless broadcast networks with elastic and inelastic traffic," in Modeling and Optimization in Mobile, Ad Hoc and Wireless Networks (WiOpt), 2011 International Symposium on. IEEE, 2011, pp. 125-132.

[5] S. Hadjiefthymiades and L. Merakos, "Using proxy cache relocation to accelerate web browsing in wireless/mobile communications," in Proceedings of the 10th international conference on World Wide Web. ACM, 2001, pp. 26-35.

[6] "C-RAN the road towards green RAN," China Mobile Research Institute, White paper, Oct. 2011.

[7] "Report on the BEREC public consultations on net neutrality," Body of European Regulators for Electronic Communications (BEREC), Report 139, Nov. 2012.

[8] M. Ji, G. Caire, and A. Molisch, "Optimal throughput-outage trade-off in wireless one-hop caching networks," in Information Theory Proceedings (ISIT), 2013 IEEE International Symposium on, July 2013, pp. 14611465.

[9] V. Jacobson, D. K. Smetters, J. D. Thornton, M. F. Plass, N. H. Briggs, and R. L. Braynard, "Networking named content," in Proceedings of the 5th international conference on Emerging networking experiments and technologies, ser. CoNEXT '09. New York, NY, USA: ACM, 2009, pp. 1-12. [Online]. Available: http://doi.acm.org/10.1145/1658939.1658941

[10] T. Koponen, M. Chawla, B.-G. Chun, A. Ermolinskiy, K. H. Kim, S. Shenker, and I. Stoica, "A data-oriented (and beyond) network architecture," in Proceedings of the 2007 conference on Applications, technologies, architectures, and protocols for computer communications, ser. SIGCOMM '07. New York, NY, USA: ACM, 2007, pp. 181-192. [Online]. Available: http://doi.acm.org/10.1145/1282380.1282402

[11] M. Gritter and D. R. Cheriton, "An architecture for content routing support in the internet," in Proceedings of the 3rd conference on USENIX Symposium on Internet Technologies and Systems - Volume 3, ser. USITS'01. Berkeley, CA, USA: USENIX Association, 2001, pp. 4-4. [Online]. Available: http://dl.acm.org/citation.cfm?id=1251440.1251444

[12] A. G. Dimakis, P. B. Godfrey, Y. Wu, M. J. Wainwright, and K. Ramchandran, "Network coding for distributed storage systems," IEEE Trans. Inf. Theory, vol. 56, no. 9, pp. 4539-4551, 2010.

[13] A. G. Dimakis, V. Prabhakaran, and K. Ramchandran, "Ubiquitous access to distributed data in large-scale sensor networks through decentralized erasure codes," in Proceedings of the 4th international symposium on Information Processing in Sensor Networks. IEEE Press, 2005 , p. 15 
[14] M. A. Maddah-Ali and U. Niesen, "Fundamental limits of caching," arXiv preprint arXiv:1209.5807, 2012.

[15] U. Niesen, D. Shah, and G. W. Wornell, "Caching in wireless networks," IEEE Trans. Inf. Theory, vol. 58, no. 10, pp. 6524-6540, 2012.

[16] E. Rosensweig and J. Kurose, "Breadcrumbs: Efficient, best-effort content location in cache networks," in INFOCOM 2009, IEEE, 2009, pp. 2631-2635.

[17] N. C. Fofack, P. Nain, G. Neglia, and D. Towsley, "Analysis of ttl-based cache networks," in Proceedings of ValueTools 2012, 2012.

[18] E. Rosensweig, J. Kurose, and D. Towsley, "Approximate models for general cache networks," in INFOCOM, 2010 Proceedings IEEE, 2010, pp. $1-9$.

[19] H. Che, Y. Tung, and Z. Wang, "Hierarchical web caching systems: modeling, design and experimental results," IEEE J.Sel. A. Commun., vol. 20, no. 7, pp. 1305-1314, Sep. 2006. [Online]. Available: http://dx.doi.org/10.1109/JSAC.2002.801752

[20] E. Rosensweig, D. Menasche, and J. Kurose, "On the steady-state of cache networks," in INFOCOM, 2013 Proceedings IEEE, 2013, pp. 19.

[21] P. Nuggehalli, V. Srinivasan, and C.-F. Chiasserini, "Energy-efficient caching strategies in ad hoc wireless networks," in Proceedings of the 4th ACM international symposium on Mobile Ad hoc Networking \& Computing. ACM, 2003, pp. 25-34.

[22] S. Jin and L. Wang, "Content and service replication strategies in multihop wireless mesh networks," in Proceedings of the 8th ACM international symposium on Modeling, Analysis and Simulation of Wireless and Mobile Systems. ACM, 2005, pp. 79-86.
[23] L. Yin and G. Cao, "Supporting cooperative caching in ad hoc networks," IEEE Transactions on Mobile Computing, vol. 5, no. 1, pp. 77-89, 2006.

[24] OpenMobileNetwork, http://map.openmobilenetwork.org/.

[25] J. Padhye, V. Firoiu, D. Towsley, and J. Kurose, "Modeling tcp throughput: A simple model and its empirical validation," in ACM SIGCOMM Computer Communication Review, vol. 28, no. 4. ACM, 1998, pp. 303-314.

[26] E. Altman, K. Avrachenkov, and C. Barakat, "A stochastic model of tcp/ip with stationary random losses," IEEE/ACM Trans. Netw., vol. 13, no. 2, pp. 356-369, Apr. 2005. [Online]. Available: http://dx.doi.org/10.1109/TNET.2005.845536

[27] T. Ho, M. Médard, R. Koetter, D. R. Karger, M. Effros, J. Shi, and B. Leong, "A random linear network coding approach to multicast," IEEE Trans. Inf. Theory, vol. 52, no. 10, pp. 4413-4430, 2006.

[28] C. Fragouli and E. Soljanin, "Network coding fundamentals," Foundations and Trends $($ in Networking, vol. 2, no. 1, pp. 1-133, 2007.

[29] M. Abramowitz and I. A. Stegun, "Handbook of mathematical functions, with formulas, graphs, and mathematical tables," 1974.

[30] A. F. Veinott, "Optimal policy in a dynamic, single product, nonstationary inventory model with several demand classes," Operations Research, vol. 13 , no. 5 , pp. 761-778, 1965.

[31] A. Müller and D. Stoyan, Comparison methods for stochastic models and risks. Wiley, 2002.

[32] F. Baccelli and B. Blaszczyszyn, Stochastic Geometry and Wireless Networks, Volume I - Theory, ser. Foundations and Trends in Networking Vol. 3: No 3-4, pp 249-449. NoW Publishers, 2009, vol. 1. 